\title{
Are We Getting Old? What Does Post Injury Mean Today?
}

Khalid Almahmoud ${ }^{*}$, Christopher Pearcy, Vaidehi Agrawal and Michael Truitt

Department of Surgery, Methodist Dallas Health System, Dallas, USA

*Corresponding author: Khalid Almahmoud, Department of Surgery, Methodist Dallas Health System, Dallas, TX 75209, USA, Tel: +4125573165; E-mail: khalidalmahmoud@mhd.com

Rec date: Aug 20, 2016; Acc date: Oct 05, 2016; Pub date: Oct 07, 2016

Copyright: ( 2016 Almahmoud K, et al. This is an open-access article distributed under the terms of the Creative Commons Attribution License, which permits unrestricted use, distribution, and reproduction in any medium, provided the original author and source are credited.

\section{Short Commentary}

Geriatric trauma patients have been identified as the fastest growing population at trauma centers across the country. By 2025, it is estimated that $40 \%$ of all trauma admits will be elderly [1]. Adults ages 65 years and older constitute the fastest-growing segment of the US population. This age group is projected to constitute $19.3 \%$ of the population by the year 2030 [2], and trauma systems are already seeing a disproportionate increase in geriatric trauma patients, as $23.7 \%$ of patients in the national trauma data bank of 2011 were 65 years and older

As these individuals present to trauma centers, several management challenges are expected given age associated physiological changes, pre-existing medical conditions (PMCs) and pre-admission medication [3]. It's crucial to develop age-specific treatment guidelines to optimize outcomes for senior trauma patients. These guidelines should emphasize the importance of field triage directly to a trauma center and the activation of the trauma team.

Furthermore, early intensive monitoring, aggressive resuscitation, and time of surgical intervention are of upmost importance to reduce mortality. Given the above noted parameters, these patients have poorer outcomes with greater risk of complication even after minor accidents [4]. Whether these outcomes are due to limited physiologic reserve, a higher incidence of PMCs, or to other factors is unclear [3]. Experimental studies in animal models can be used to mimic the trauma response post injury in geriatric population, and to translate these finding to the clinical practice. In this short review, we summarize the cause of injury in this population, the impact of various risk factors associated with poor outcomes and the various guidelines that have been instated to assist in the management of these patient for improved improve morbidity and mortality.

The dominant cause of injury among the geriatric patients greater than 65 years is predominantly falls $[5,6]$. Sterling et al. demonstrated that $48 \%$ of the patients who are older than 65 years presented after falls compared to only $7 \%$ in patients younger than 65 . Additionally, it has also been reported that elderly patients had a higher injury severity after falls in comparison to their younger cohort. The increased severity score was largely due to the higher incidence of head injury, intracranial injuries, pelvic fracture, femoral fracture, and spinal fracture involvement; however, there was no significant difference in solid or hollow viscose organs involvement between the groups $[7,8]$. Upon admission of the patient to the care facility, several risk factors have been previously demonstrated to be associated with poor outcomes among the elderly.

The first and foremost significant contributor to high post-traumatic mortality is age. Although no censes has been reached on the cut-off for mortality in geriatric patients, several studies describe a steady increase in mortality with increasing age $[1,4]$. Some publications indicate that age of greater than 55 years is associated with higher risk of death, while others report that an age of 65 years is associated with an increase in death rate at twice that of a younger patient population $[6,7]$. PMCs have also been described to be of major importance for clinical course in geriatric trauma patients.

PMCs are frequently found in the elderly population. In this context, an incidence of PMC of more than $33 \%$ in trauma patients aged older than 75 years has been described, compared to $3.5 \%$ in the younger population [6]. The presence of PMCs can significantly compromise an elderly trauma victim's ability to compensate, as well as recover from severe injury [4]. Accordingly, geriatric trauma patients with PMCs were shown to have worse outcomes when compared to patients without major co-morbidities. Therefore, incorporating information about PMCs is essential for mortality analysis in the elderly trauma patient. In addition to PMCs, preadmission medication use has also been described to affect the posttraumatic course.

Besides PMCs, premeditations use has been described to affect the post-traumatic course. Several studies described an independent association between the use of anticoagulation medication and the incidence of traumatic intracranial hemorrhage with a reported 2-10fold increase in risk of death among geriatric patients [6]. To our knowledge, other medications have not been evaluated for association with poor outcomes in this population.

Furthermore, controversial discussion on the effects of beta blockers use on the post-traumatic course were reported, as beta blockers have been associated with increased post-traumatic mortality rate, through hiding the shock states or decrease the body's natural response to trauma, which may lead to an extended period of suboptimal or under-resuscitation [9]. On the other hand, other studies demonstrated a beneficial effect for the beta blockers in trauma patients with head injuries by reducing the metabolic rates in brain tissues [10]. Thus, further work is needed to asses if PMCs and medications use are independent risk factors.

The impact of the above factors has also been demonstrated to be linked with altered short term outcomes in this patient population. One study demonstrated that elderly patients ( $>65$ years) with severe trauma (ISS $>30$ ) have been reported to have a decreased ICU admission rates [6]. Contrarily, another study demonstrated a higher ICU admission rate due to the propensity of rapid deterioration in geriatric trauma patients [6]. This conflict in the results might be explained by the study design (e.g. in- or exclusion of patients unlikely to survive) and the impact of other trauma-associated variables (mechanism of injury, injury severity, and distribution). In the general population, age was identified as a risk factor in one-year and longterm survival after intensive care treatment in patients older than 65 years [6-8]. 
Page 2 of 2

Several studies have associated increasing patient age with a higher incidence of post-traumatic complications $[1,6,11]$. In this context, a complication rate of up to $50 \%$ in elderly trauma patients over the clinical course has been described. The complications include infections (pneumonia, urinary tract infection), thromboembolic incidents and organ failure $[1,11,12]$.

Following injury, inflammatory responses differ between young adult and aged subjects. Valente et al. [13] investigated the innate immune function, and found significant increase in CD18 levels, and a higher severity of the oxidative burst reaction in the geriatric trauma group in the later phase. Also, he assumed that neutrophils from injured elderly patients either might require a longer time to mount a response or that their inflammatory response to trauma is prolonged [13].

These findings are supported by earlier investigations on the immune response to infections, as cytokine release in the elderly patients ( $>68$ years) compared to younger patients ( $<55$ years) is limited to pro-inflammatory activation, but accompanied by prolonged anti-inflammatory activity over one week $[14,15]$. Aging has also been assumed to have an effect on T-cells with a replacement of virgin cells by memory cells, and an accumulation of cells with signal transduction defects [16]. In addition, a decreased cytokine response and a decreased incidence of fever have been observed as signs of an impaired immune response [17]. As one possible mechanism for the impairment of immune function, the higher incidence of posttraumatic hyperglycemia in elderly patients has been suggested [18-20].

In terms of management, elderly patients benefit from both early admissions to a trauma center with geriatric trauma experience and immediate trauma team activation. Outcomes can be improved by subtle monitoring, aggressive resuscitation, and surgical management which is adapted to the physiological reserves of the patient. However, timely estimation of the risk profile of elderly patients might be aggravated due to a blunted physiologic response. There is some evidence from the literature that age-associated physiologic, immunologic, and metabolic changes contribute to the different clinical course in geriatric trauma patients. A better understanding of the pathophysiological mechanisms is needed to develop age-specific therapies through more investigation in future studies.

\section{Conclusion}

Treatment of geriatric patients after severe trauma requires the attention of different age-specific considerations. Yet, the exact therapeutic and diagnostic requirements for elderly trauma patients are not fully addressed in the current trauma system; therefore, it is crucial for trauma centers to develop specific management guidelines for this population. In addition, having age-specific management guidelines in trauma centers will improve the outcomes in this group of patients. Finally, to identify a potential starting point for new therapeutic strategies, the specific impact of numerous factors (age, incidence of PMCs, decreased physiologic reserves, and impaired immune function) on outcome differences between the elderly and the younger trauma population need to be further clarified through experimental and clinical studies in the near future.

\section{References}

1. Richmond TS, Kauder D, Strumpf N, Meredith T (2002) Characteristics and outcomes of serious traumatic injury in older adults. J Am Geriatr Soc 50: 215-222.

2. Goodmanson NW, Rosengart MR, Barnato AE, Sperry JL, Peitzman AB, et al. (2012) Defining geriatric trauma: when does age make a difference? Surgery 152: 668-674.

3. Bala M, Willner D, Klauzni D, Bdolah-Abram T, Rivkind AI, et al. (2013) Pre-hospital and admission parameters predict in-hospital mortality among patients 60 years and older following severe trauma. Scand J Trauma Resusc Emerg Med 21:91.

4. Gowing R, Jain MK (2007) Injury patterns and outcomes associated with elderly trauma victims in Kingston, Ontario. Can J Surg 50: 437-444.

5. Sampalis JS, Nathanson R, Vaillancourt J, Nikolis A, Liberman M, et al. (2009) Assessment of mortality in older trauma patients sustaining injuries from falls or motor vehicle collisions treated in regional level I trauma centers. Ann Surg 249: 488-495.

6. Hannan EL, Waller CH, Farrell LS, Rosati C (2004) Elderly trauma inpatients in New York state: 1994-1998. J Trauma 56: 1297-1304.

7. Spaniolas K, Cheng JD, Gestring ML, Sangosanya A, Stassen NA, et al. (2010) Ground level falls are associated with significant mortality in elderly patients. J Trauma 69: 821-825.

8. Demetriades D, Murray J, Brown C, Velmahos G, Salim A, et al. (2005) High-level falls: type and severity of injuries and survival outcome according to age. J Trauma 58: 342-345.

9. Neideen T, Lam M, Brasel KJ (2008) Preinjury beta blockers are associated with increased mortality in geriatric trauma patients. J Trauma 65: 1016-1020.

10. Cotton BA, Snodgrass KB, Fleming SB, Carpenter RO, Kemp CD, et al. (2007) Beta-blocker exposure is associated with improved survival after severe traumatic brain injury. J Trauma 62: 26-33.

11. Adams SD, Cotton BA, McGuire MF, Dipasupil E, Podbielski JM, et al. (2012) Unique pattern of complications in elderly trauma patients at a Level I trauma center. J Trauma Acute Care Surg. 72: 112-118.

12. Hannan EL, Farrell LS, Gorthy SF, Bessey PQ, Cayten CG, et al. (1999) Predictors of mortality in adult patients with blunt injuries in New York State: a comparison of the trauma and injury severity score (TRISS) and the international classification of disease, ninth revision-based injury severity score (ICISS). J Trauma 47: 8-14.

13. Valente SA, Fallon WF, Alexander TS, Tomas ER, Evancho-Chapman MM, et al. (2009) Immunologic function in the elderly after injury--the neutrophil and innate immunity. J Trauma 67: 968-974.

14. Bruunsgaard H, Skinhoj P, Qvist J, Pedersen BK (1999) Elderly humans show prolonged in vivo inflammatory activity during pneumococcal infections. J Infect Dis 180: 551-554.

15. Bruunsgaard H, Pedersen $M$, Pedersen BK (2001) Aging and proinflammatory cytokines. Curr Opin Hematol 8: 131-136.

16. Miller RA (1996) The aging immune system: primer and prospectus. Science 273: 70-74.

17. Frankenfield D, Cooney RN, Smith JS, Rowe WA (2000) Age-related differences in the metabolic response to injury. J Trauma 48: 49-56.

18. Almahmoud K, Namas RA, Abdul-Malak O, Zaaqoq AM, Zamora R, et al. (2015) Impact of injury severity on dynamic inflammation networks following blunt trauma. Shock 44: 101-109.

19. Nagy KK, Smith RF, Roberts RR, Joseph KT, An GC, et al. (2000) Prognosis of penetrating trauma in elderly patients: a comparison with younger patients. The J Trauma 49: 190-193.

20. Salottolo KM, Mains CW, Offner PJ, Bourg PW, Bar-Or D (2013) A retrospective analysis of geriatric trauma patients: venous lactate is a better predictor of mortality than traditional vital signs. Scand J Trauma Resusc Emerg Med 21: 7. 